PROCEEDINGS OF THE

AMERICAN MATHEMATICAL SOCIETY

Volume 138, Number 10, October 2010, Pages 3425-3436

S 0002-9939(2010)10494-X

Article electronically published on May 20, 2010

\title{
FROBENIUS GROUPS AS GROUPS OF AUTOMORPHISMS
}

\author{
N. YU. MAKARENKO AND PAVEL SHUMYATSKY
}

(Communicated by Jonathan I. Hall)

\begin{abstract}
We show that if GFH is a double Frobenius group with "upper" complement $H$ of order $q$ such that $C_{G}(H)$ is nilpotent of class $c$, then $G$ is nilpotent of $(c, q)$-bounded class. This solves a problem posed by Mazurov in the Kourovka Notebook. The proof is based on an analogous result on Lie rings: if a finite Frobenius group $F H$ with kernel $F$ of prime order and complement $H$ of order $q$ acts on a Lie ring $K$ in such a way that $C_{K}(F)=0$ and $C_{K}(H)$ is nilpotent of class $c$, then $K$ is nilpotent of $(c, q)$-bounded class.
\end{abstract}

\section{INTRODUCTION}

The situation where a Frobenius group acts by automorphisms on another group or a ring has recently received some study. Recall that a Frobenius group FH with kernel $F$ and complement $H$ can be characterized as a finite group that is a semidirect product of a normal subgroup $F$ on which $H$ acts by automorphisms so that $C_{F}(h)=1$ for every $h \in H \backslash\{1\}$. In particular, $H$ acts faithfully on every non-trivial $H$-invariant subgroup of $F$. It follows that all abelian subgroups of $H$ are cyclic. By Thompson's theorem 13 the kernel $F$ is nilpotent, and by Higman's theorem [4 the nilpotency class of $F$ is bounded in terms of the least prime divisor of $|H|$ (explicit upper bounds for the nilpotency class are due to Kreknin and Kostrikin [9, 10]).

If a Frobenius group $F H$ acts on a group $G$ in such a manner that $G F$ is also a Frobenius group, then the product $G F H$ is called a double Frobenius group. The double Frobenius groups arise, in particular, in the study of the Gruenberg-Kegel prime graphs of finite groups. In [11] Mazurov proved that if $G F H$ is a double Frobenius group such that $C_{G}(H)$ is abelian and $H$ is of order 2 or 3 , then $G$ is nilpotent of class at most 2 . In view of this result he raised the question whether in general the nilpotency class of $G$ can be bounded in terms of $|H|$ and the class of $C_{G}(H)$ only (see [8, Problem 17.72(a)]). The first substantial evidence that the answer to the question might be positive was obtained by Khukhro. He proved in [7] that if $(|G|,|H|)=1$ and $C_{G}(H)$ is abelian, then $G$ is nilpotent of class bounded solely in terms of $|H|$. The purpose of the present paper is to confirm that the

Received by the editors November 13, 2009.

2010 Mathematics Subject Classification. Primary 20D45, 17B70.

Key words and phrases. Automorphisms, centralizers, associated Lie rings.

The first author was supported in part by the Programme of Support of Leading Scientific Schools of the Russian Federation.

The second author was supported by CNPq-Brazil. 
answer to Mazurov's question is positive. In particular, we prove the following theorem.

Theorem 1.1. Let GFH be a double Frobenius group with complement $H$ of order q. Assume that $C_{G}(H)$ is nilpotent of class $c$. Then $G$ is nilpotent of $(c, q)$-bounded class.

It should be said that in the original version of the paper the above theorem was proved under the additional hypothesis that $(|G|, q)=1$. This, of course, would give only a partial answer to Mazurov's question. Luckily for us, Khukhro suggested an idea that enabled us to remove the assumption that $(|G|, q)=1$ from the hypothesis of the theorem. Both Lemma 3.2 and Lemma 3.3 in Section 3 are due to Khukhro. We would like to thank him for the help.

Theorem 1.1 is deduced from a corresponding result on Lie rings. In Section 3 we prove that if $F H$ is a Frobenius group with kernel $F$ of prime order and complement $H$ of order $q$ that acts by automorphisms on a Lie ring $K$ in such a way that $C_{K}(F)=0$ and $C_{K}(H)$ is nilpotent of class $c$, then $K$ is nilpotent of $(c, q)$-bounded class (Theorem 3.1). In turn, Theorem 3.1 is deduced from a rather complicated criterion of nilpotency for graded Lie algebras obtained in Section 2. We make no attempts to write down an explicit bound for the class of the group $G$ in Theorem 1.1 but the reader can easily check that working through the proofs one can indeed obtain a bound as a function of $c$ and $q$. Throughout the paper we use the expression " $(a, b, c, \ldots)$-bounded" to mean "bounded from above by some function depending only on $a, b, c, \ldots$ ".

\section{A COMBINATORIAL CONDITION ON GRADED LIE ALGEBRAS}

In this section $q$ stands for an arbitrary but fixed positive integer. Choose a prime $p$ such that $q$ divides $p-1$ and a positive integer $r \leq p-1$ such that $r$ has multiplicative order $q$ modulo $p$. Thus, $r^{q} \equiv 1(\bmod p)$. When convenient, we freely identify $r$ with its image in $\mathbb{Z} / p \mathbb{Z}$ and regard $r$ as an element of $\mathbb{Z} / p \mathbb{Z}$ such that $r^{q}=1$. We will work with finite sequences of non-zero elements of $\mathbb{Z} / p \mathbb{Z}$. Let $a_{1}, \ldots, a_{k}$ be not necessarily distinct non-zero elements in $\mathbb{Z} / p \mathbb{Z}$. We say that the sequence $\left(a_{1}, \ldots, a_{k}\right)$ is $r$-dependent if and only if there exist distinct $i_{1}, \ldots, i_{m} \in\{1,2, \ldots, k\}$ and not necessarily distinct $\alpha_{1}, \ldots, \alpha_{m} \in\{1,2, \ldots, q-1\}$ such that

$$
a_{i_{1}}+\cdots+a_{i_{m}}=r^{\alpha_{1}} a_{i_{1}}+\cdots+r^{\alpha_{m}} a_{i_{m}} .
$$

If the sequence $\left(a_{1}, \ldots, a_{k}\right)$ is not $r$-dependent, we will call it $r$-independent. We denote by $F_{k}$ the set of all $r$-independent sequences of length $k$ and by $Z_{k}$ the set of all $r$-dependent ones.

For a given sequence $\left(d_{1}, \ldots, d_{k}\right) \in F_{k}$ we denote by $M\left(d_{1}, \ldots, d_{k}\right)$ the set of all $j \in \mathbb{Z} / p \mathbb{Z}$ such that $\left(d_{1}, \ldots, d_{k}, j\right) \in Z_{k+1}$.

Lemma 2.1. If $\left(d_{1}, \ldots, d_{k}\right) \in F_{k}$, then $\left|M\left(d_{1}, \ldots, d_{k}\right)\right| \leq q^{k+1}$.

Proof. Suppose that $\left(d_{1}, \ldots, d_{k}, j\right) \in Z_{k+1}$. We have

$$
d_{1}+d_{2}+\cdots+d_{k}+j=r^{i_{1}} d_{1}+r^{i_{2}} d_{2}+\cdots+r^{i_{k}} d_{k}+r^{i_{0}} j
$$

for suitable $0 \leq i_{s} \leq q-1$, where not all of the $i_{s}$ equal 0 . In fact, $i_{0} \neq 0$ for otherwise the sequence $\left(d_{1}, \ldots, d_{k}\right)$ would not be $r$-independent. We see that

$$
j=\left(r^{i_{1}} d_{1}+\cdots+r^{i_{k}} d_{k}-d_{1}-d_{2}+\cdots-d_{k}\right) /\left(1-r^{i_{0}}\right),
$$


so there are at most $q^{k+1}$ possibilities for $j$, as required.

In the next two lemmas we will require some facts on polynomials.

Lemma 2.2. Let $K$ be a field of characteristic 0 containing a primitive qth root of unity $\omega$. Suppose that for some positive integer $m$ and some $0 \leq i_{1}, \ldots, i_{m} \leq q-1$ we have $m=\omega^{i_{1}}+\cdots+\omega^{i_{m}}$. Then $i_{1}=\cdots=i_{m}=0$.

Proof. Without loss of generality it can be assumed that $K=\mathbb{Q}[\omega]$. Since $m=$ $\left|\omega^{i_{1}}\right|+\cdots+\left|\omega^{i_{m}}\right|$, the lemma follows from the triangle inequality.

Lemma 2.3. If the sequence $\left(a_{1}, \ldots, a_{n}\right)$ is $r$-dependent and $a_{1}=\cdots=a_{n}$, then $p \leq n^{q-1}(2 q-2)$ !.

Proof. Suppose that $a_{1}=\cdots=a_{n}$. In this case the $r$-dependence of $\left(a_{1}, \ldots, a_{n}\right)$ means that for some $m \leq n$ and some $1 \leq i_{1}, \ldots, i_{m} \leq q-1$ we have $m=$ $r^{i_{1}}+\cdots+r^{i_{m}}$. Therefore $r$ is a root of two polynomials

$$
\begin{gathered}
x^{q-1}+\cdots+x^{1}+1, \\
b_{q-1} x^{q-1}+\cdots+b_{1} x-m,
\end{gathered}
$$

where $\sum_{i=1}^{q-1} b_{i}=m$. When convenient we will somewhat abuse the notation and regard the polynomials as ones over $\mathbb{Z}$. In that case we assume that $b_{i} \in\{0,1, \ldots, m\}$. The two polynomials have a common root if and only if their resultant is zero. The resultant is the determinant of the Sylvester matrix consisting of the coefficients of the polynomials. Thus, the polynomials have a common root in $\mathbb{Z} / p \mathbb{Z}$ if

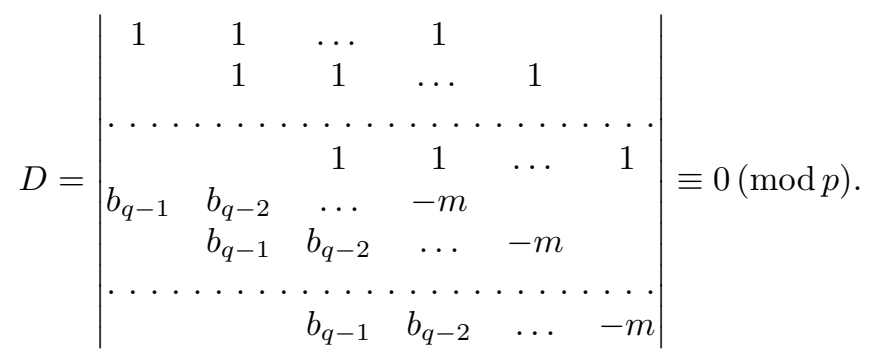

(Here the matrix has $2 q-2$ lines: $q-1$ lines correspond to the first polynomial and $q-1$ to the second one. The empty part of the matrix is filled with zeros.) By Lemma 2.2 the polynomials have no common roots in a field of characteristic zero. Hence, $D$ cannot be equal to 0 . Since $b_{i} \in\{0,1, \ldots, m\}$, we check that $D \leq n^{q-1}(2 q-2)$ ! (a very rough estimate). The prime $p$ must divide $D$ since the resultant is zero in $\mathbb{Z} / p \mathbb{Z}$. Therefore $p \leq n^{q-1}(2 q-2)$ !.

Next, we will give a sufficient condition to conclude that the sequence $\left(a_{1}, \ldots, a_{n}\right)$ contains an $r$-independent subsequence.

Lemma 2.4. Suppose that for some $m$ the sequence $\left(a_{1}, \ldots, a_{n}\right)$ contains at least $q^{m}+m$ different values. Then one can choose an $r$-independent subsequence $\left(a_{1}, a_{i_{2}}, \ldots, a_{i_{m}}\right)$ of $m$ elements that contains $a_{1}$.

Proof. If $m=1$, the lemma is obvious, so we assume that $m \geq 2$ and use induction on $m$. By induction we can choose an $r$-independent subsequence $\left(a_{1}, a_{i_{2}}, \ldots, a_{i_{m-1}}\right)$ of $m-1$ elements. Without loss of generality it can be assumed that $i_{2}=$ $2, \ldots, i_{m-1}=m-1$. Thus, $\left(a_{1}, a_{2}, \ldots, a_{m-1}\right)$ is an $r$-independent subsequence. By Lemma 2.1 there are at most $q^{m}$ distinct elements in $M\left(a_{1}, a_{2}, \ldots, a_{m-1}\right)$. The 
hypothesis tells us that the sequence $a_{1}, \ldots, a_{n}$ contains at least $q^{m}+m$ different values. Therefore the sequence $a_{m}, a_{m+1} \ldots, a_{n}$ contains at least $q^{m}+1$ different values. So we can always choose $b$ among $a_{m}, a_{m+1} \ldots, a_{n}$ such that the sequence $a_{1}, a_{2}, \ldots, a_{m-1}, b$ is $r$-independent.

Let $A$ be an additively written abelian group. A Lie $\operatorname{ring} L$ is $A$-graded if

$$
L=\bigoplus_{a \in A} L_{a}, \quad \text { and } \quad\left[L_{a}, L_{b}\right] \subseteq L_{a+b},
$$

where the $L_{a}$ are subgroups of the additive group of $L$. Elements of the grading components $L_{a}$ are called homogeneous, and commutators in homogeneous elements homogeneous commutators. An additive subgroup $H$ of $L$ is called homogeneous if $H=\bigoplus_{a}\left(H \cap L_{a}\right)$; we then write $H_{a}=H \cap L_{a}$. Clearly, any subring or ideal generated by homogeneous additive subgroups is homogeneous. A homogeneous subring and the quotient by a homogeneous ideal can be regarded as $A$-graded Lie rings with the induced gradings. Also, it is not difficult to see that if $H$ is homogeneous, then so is $C_{L}(H)$. Here the centralizer of $H$ is, as usual, the set $\{l \in L \mid[l, h]=0$ for all $h \in H\}$. We denote by ${ }_{i d}\langle S\rangle$ the ideal generated by the set $S$.

Throughout this section we will work with a $\mathbb{Z} / p \mathbb{Z}$-graded Lie ring $L$ such that $L_{0}=0$. To avoid the overloaded notation we make the following convention.

Index Convention. Henceforth a small Latin letter with an index $i \in \mathbb{Z} / p \mathbb{Z}$ will denote a homogeneous element in the grading component $L_{i}$, with the index only indicating which component this element belongs to: $x_{i} \in L_{i}$. We will not be using numbering indices for elements of the $L_{i}$, so that different elements can be denoted by the same symbol when it only matters which component the elements belong to. For example, $x_{i}$ and $x_{i}$ can be different elements of $L_{i}$, so that $\left[x_{i}, x_{i}\right]$ can be a non-zero element of $L_{2 i}$.

Note that under the index convention a homogeneous commutator belongs to the component $L_{s}$, where $s$ is the sum of the indices of all the elements involved in this commutator.

In what follows we assume that there exists a non-negative integer $c$ such that the ring $L$ satisfies the condition that

$$
\left[x_{d_{1}}, x_{d_{2}}, \ldots, x_{d_{c+1}}\right]=0 \text { whenever }\left(d_{1}, \ldots, d_{c+1}\right) \in F_{c+1} .
$$

The main goal of this section is to prove that the condition (2.1) is sufficient to conclude that $L$ is nilpotent of $(c, q)$-bounded class (see Theorem 2.13 in the end of the section).

Lemma 2.5. For each $b \in \mathbb{Z} / p \mathbb{Z}$ there are at most

$$
\max \left\{q^{c+1}, c^{q-1}(2 q-2) !\right\}
$$

elements $a \in \mathbb{Z} / p \mathbb{Z}$ such that $[L_{a}, \underbrace{L_{b}, \ldots, L_{b}}_{c}] \neq 0$.

Proof. Suppose that $\left[L_{a}, L_{b}, \ldots, L_{b}\right] \neq 0$. Then the sequence $(a, \underbrace{b, \ldots, b}_{c})$ belongs to $Z_{c+1}$ and so we have $a+b+\cdots+b=r^{i_{0}} a+r^{i_{1}} b+\cdots+r^{i_{c}} b$ for suitable $0 \leq i_{j} \leq q-1$, where at least once $i_{j} \neq 0$. If $i_{0} \neq 0$, we see that

$$
a=\left(r^{i_{1}} b+\cdots+r^{i_{c}} b-c b\right) /\left(1-r^{i_{0}}\right),
$$


so there are at most $q^{c+1}$ possibilities for $a$. If $i_{0}=0$, then for some $1 \leq m \leq c$ and some $1 \leq j_{1}, \ldots, j_{m} \leq q-1$ we have $m=r^{j_{1}}+\cdots+r^{j_{m}}$. In this case, by Lemma 2.3, $p \leq c^{q-1}(2 q-2)$ ! and the result follows.

Lemma 2.6. There exists a $(c, q)$-bounded number $w$ such that for all $a, b \in \mathbb{Z} / p \mathbb{Z}$ we have $[L_{a}, \underbrace{L_{b}, \ldots, L_{b}}_{w}]=0$.

Proof. Given $b \in \mathbb{Z} / p \mathbb{Z}$, we denote by $N(b)$ the set of all $a \in \mathbb{Z} / p \mathbb{Z}$ such that $[L_{a}, \underbrace{L_{b}, \ldots, L_{b}}_{c}] \neq 0$. According to Lemma 2.5. $N=|N(b)| \leq \max \left\{q^{c+1}\right.$, $\left.c^{q-1}(2 q-2) !\right\}$. If for some $t \geq 1$ we have $[L_{a}, \underbrace{L_{b}, \ldots, L_{b}}_{c+t}] \neq 0$, then all elements $a, a+b, a+2 b, a+t b$ belong to $N(b)$. It follows that either $[L_{a}, \underbrace{L_{b}, \ldots, L_{b}}_{c+N}]=0$ for all $a \in \mathbb{Z} / p \mathbb{Z}$ or $t b=0$ for some $t \leq N$. In the latter case we conclude that $p \leq N$. Clearly, for all $a, b$ there exists an integer $s$ such that $0 \leq s \leq p-1$ and $a+s b=0$. Therefore $[L_{a}, \underbrace{L_{b}, \ldots, L_{b}}_{N-1}]=0$, and so the lemma follows.

Choose $\left(d_{1}, \ldots, d_{c}\right) \in F_{c}$ and fix a commutator $U=\left[u_{d_{1}}, \ldots, u_{d_{c}}\right]$.

Lemma 2.7. Every commutator of the form

$$
\left[U, x_{i_{1}}, \ldots, x_{i_{t}}\right]
$$

can be written as a linear combination of commutators of the form

$$
\left[U, m_{j_{1}}, \ldots, m_{j_{h}}\right]
$$

where $j_{s} \in M\left(d_{1}, \ldots, d_{c}\right)$ and $h \leq t$. The case $t=h$ is possible only if $i_{s} \in$ $M\left(d_{1}, \ldots, d_{c}\right)$ for all $s=1, \ldots, t$.

Proof. The assertion is obviously true for $t=0$. Let $t=1$. If $i_{1} \in M\left(d_{1}, \ldots, d_{c}\right)$, then $\left[U, x_{i_{1}}\right]$ is of the required form. If $i_{1} \notin M\left(d_{1}, \ldots, d_{c}\right)$, then $\left[U, x_{i_{1}}\right]=0$ by $(2.1)$ and there is nothing to prove.

Let us assume that $t>1$ and use induction on $t$. If all the indices $i_{j}$ belong to $M\left(d_{1}, \ldots, d_{c}\right)$, then the commutator $\left[U, x_{i_{1}}, \ldots, x_{i_{t}}\right]$ is of the required form with $h=t$. Suppose that in (2.2) there is an element $x_{i_{k}}$ with the index $i_{k}$ that does not belong to $M\left(d_{1}, \ldots, d_{c}\right)$. We choose such an element with $k$ as small as possible and use $k$ as a second induction parameter.

If $k=1$, the commutator (2.2) is zero and we are done. Suppose that $k \geq 2$ and write

$$
\begin{aligned}
& {\left[U, \ldots, x_{i_{k-1}}, x_{i_{k}}, \ldots, x_{i_{t}}\right]} \\
& \quad=\left[U, \ldots, x_{i_{k}}, x_{i_{k-1}}, \ldots, x_{i_{t}}\right]+\left[U, \ldots,\left[x_{i_{k-1}}, x_{i_{k}}\right], \ldots, x_{i_{t}}\right] .
\end{aligned}
$$

The induction hypothesis forces us to conclude that the commutator $[U, \ldots$, $\left.\left[x_{i_{k-1}}, x_{i_{k}}\right], \ldots, x_{i_{t}}\right]$ is a linear combination of commutators of the form (2.3) because it is shorter than $(2.2)$, while the commutator $\left[U, \ldots, x_{i_{k}}, x_{i_{k-1}}, \ldots, x_{i_{t}}\right]$ is a linear combination of commutators of the form (2.3) because the index that does not belong to $M\left(d_{1}, \ldots, d_{c}\right)$ here occurs closer to $U$ than in (2.2). This completes the proof. 
Corollary 2.8. The ideal of $L$ generated by $U$ is spanned by commutators of the form (2.3).

Let $M=\left|M\left(d_{1}, \ldots, d_{c}\right)\right|$ and let $w$ be from Lemma 2.6. In the next lemma we obtain a rather detailed information about the ideal generated by $U$.

Lemma 2.9. ${ }_{i d}\langle U\rangle$ is spanned by those commutators of the form (2.3) for which $h \leq(w-1) M$.

Proof. Let $R$ be the linear span of the commutators of the form (2.3) for which $h \leq(w-1) M$. It is sufficient to show that every commutator of the form (2.3) belongs to $R$. So choose a commutator $W=\left[U, m_{j_{1}}, \ldots, m_{j_{h}}\right]$ of the form (2.3). If $h \leq(w-1) M$, it is clear that $W \in R$, so we assume that $h>(w-1) M$ and use induction on $h$. Write

$$
W=\left[U, m_{j_{1}}, \ldots, m_{j_{t}}, m_{j_{t-1}}, \ldots, m_{j_{h}}\right]+\left[U, m_{j_{1}}, \ldots,\left[m_{j_{t-1}}, m_{j_{t}}\right], \ldots, m_{j_{h}}\right] .
$$

If $j_{t-1}+j_{t} \in M\left(d_{1}, \ldots, d_{c}\right)$, then the second summand is of the form (2.3). Since it is shorter than $W$, it belongs to $R$ by the induction hypothesis. If $j_{t-1}+j_{t} \notin$ $M\left(d_{1}, \ldots, d_{c}\right)$, we apply Lemma 2.7 and conclude that the second summand can be written as a linear combination of commutators of the form (2.3) each of which is shorter than $W$. Thus, in either case $\left[U, m_{j_{1}}, \ldots,\left[m_{j_{t-1}}, m_{j_{t}}\right], \ldots, m_{j_{h}}\right] \in R$.

It follows that the commutator $W$ does not change modulo $R$ under any permutation of the $m_{j_{k}}$. By our assumption, the number $h$ is big enough to guarantee that among the elements $m_{j_{k}}$ there are at least $w$ that belong to the same component $L_{i}$. Since $W$ does not change modulo $R$ under any permutation of the $m_{j_{k}}$, without loss of generality we can assume that $m_{j_{1}}, \ldots, m_{j_{w}} \in L_{i}$. But now it follows from Lemma 2.6 that $W=0$. This completes the proof.

Corollary 2.10. The ideal ${ }_{i d}\left\langle\left[L_{d_{1}}, \ldots, L_{d_{c}}\right]\right\rangle$ has $(c, q)$-boundedly many non-trivial components of the induced grading.

Proof. Since in $(2.3) j_{i} \in M\left(d_{1}, \ldots, d_{c}\right)$ for all $i=1, \ldots, h$, the sum of all indices in (2.3) can take at most $M^{h}$ values. By Lemma 2.1 and Lemma 2.6 the number $h$ is $(c, q)$-bounded. So ${ }_{i d}\langle U\rangle$ has $(c, q)$-boundedly many non-trivial components. It follows from the proofs of Lemma 2.9 and Lemma 2.7 that the set of all possible non-trivial components in ${ }_{i d}\langle U\rangle$ is completely determined by the tuple $\left(d_{1}, \ldots, d_{c}\right)$ and does not depend on the choice of $U=\left[u_{d_{1}}, \ldots, u_{d_{c}}\right]$. Since the ideal ${ }_{i d}\left\langle\left[L_{d_{1}}, \ldots, L_{d_{c}}\right]\right\rangle$ is the sum of ideals ${ }_{i d}\left\langle\left[u_{d_{1}}, \ldots, u_{d_{c}}\right]\right\rangle$ over all possible $u_{d_{1}}, \ldots, u_{d_{c}}$, the result follows.

Lemma 2.11. Suppose that a homogeneous ideal $T$ of $L$ has only e non-trivial components. Then $L$ has at most $e^{2}$ components that do not centralize $T$.

Proof. Let $T_{i_{1}}, \ldots, T_{i_{e}}$ be the non-trivial homogeneous components of $T$ and let $S=\left\{i_{1}, \ldots, i_{e}\right\}$. Suppose that $L_{i}$ does not centralize $T$. Then for some $j \in S$ we have $i+j \in S$. So there are at most $|S| \times|S|$ possibilities for $i$, as required.

We are now almost ready to prove the main result of this section. In the next proposition we will establish that $L$ is soluble with bounded derived length. After that it will be easy to deduce that $L$ is nilpotent of bounded class.

Proposition 2.12. The ring $L$ is soluble with $(c, q)$-bounded derived length. 
Proof. If $c=0$, then $L=0$ by (2.1) and there is nothing to prove. Assume that $c \geq 1$ and use induction on $c$. Let $I$ be the ideal of $L$ generated by all commutators $\left[L_{i_{1}}, \ldots, L_{i_{c}}\right]$, where $\left(i_{1}, \ldots, i_{c}\right)$ ranges through $F_{c}$. The induction hypothesis will be that $L / I$ is soluble with bounded derived length, say $f_{0}$.

Choose $\left(i_{1}, \ldots, i_{c}\right) \in F_{c}$ and look at the ideal $T={ }_{i d}\left\langle\left[L_{i_{1}}, \ldots, L_{i_{c}}\right]\right\rangle$. We know from Corollary 2.10 that there are only $(c, q)$-boundedly many, say $e$, non-trivial grading components in $T$. By Lemma 2.11 there are at most $e^{2}$ components that do not centralize $T$. Since $C_{L}(T)$ is also a homogeneous ideal, it follows that the quotient $L / C_{L}(T)$ has at most $e^{2}$ non-trivial components. By a theorem of Shalev 12 we conclude that $L / C_{L}(T)$ is soluble with $e$-bounded derived length, say $f_{1}$. Therefore $L^{\left(f_{1}\right)}$, the corresponding term of the derived series, centralizes $T$. Since $f_{1}$ does not depend on the choice of the tuple $\left(i_{1}, \ldots, i_{c}\right) \in F_{c}$, it follows that $\left[L^{\left(f_{1}\right)}, I\right]=0$. Recall that $L^{\left(f_{0}\right)} \leq I$. Hence, $\left[L^{\left(f_{1}\right)}, L^{\left(f_{0}\right)}\right]=0$. From this we deduce that $L$ is soluble with derived length at $\operatorname{most} \max \left\{f_{0}, f_{1}\right\}+1$. The proof is complete.

Theorem 2.13. The ring $L$ is nilpotent of $(c, q)$-bounded class.

Proof. We know from Proposition 2.12 that $L$ is soluble of $(c, q)$-bounded derived length. Let us use induction on the derived length of $L$. If $L$ is abelian, there is nothing to prove. Assume that $L$ is metabelian. In this case $[x, y, z]=[x, z, y]$ for every $x \in[L, L]$ and $y, z \in L$. For each $a \in \mathbb{Z} / p \mathbb{Z}$ we denote $[L, L] \cap L_{a}$ by $L_{a}^{\prime}$. Let $w=w(c, q)$ be as in Lemma 2.6 and put $n=(w-1)\left(q^{c+1}+c\right)+2$. Choose a sequence of $n$ elements $\left(a_{1}, \ldots, a_{n}\right)$ in $\mathbb{Z} / p \mathbb{Z}$ and consider the commutator $\left[L_{a_{1}}^{\prime}, L_{a_{2}}, \ldots, L_{a_{n}}\right]$. Assume that $\left[L_{a_{1}}^{\prime}, L_{a_{2}}, \ldots, L_{a_{n}}\right] \neq 0$. Suppose that $\left(a_{1}, \ldots, a_{n}\right)$ contains an $r$-independent subsequence of length $c+1$ that starts with $a_{1}$. Using that $[x, y, z]=[x, z, y]$ we can assume that $a_{1}, \ldots, a_{c+1}$ is the $r$-independent subsequence. Thus, by (2.1) the commutator $\left[L_{a_{1}}^{\prime}, L_{a_{2}}, \ldots, L_{a_{n}}\right]$ is zero, a contradiction. It follows from Lemma 2.4 that the sequence $a_{1}, \ldots, a_{n}$ contains at most $q^{c+1}+c$ different values. The number $n$ was chosen big enough to guarantee that either the value of $a_{1}$ occurs in $a_{1}, \ldots, a_{n}$ at least $w+1$ times or else, another value, different from $a_{1}$, occurs at least $w$ times. Using that $[x, y, z]=[x, z, y]$ we can assume that $a_{2}=\cdots=a_{w}$, in which case it follows from Lemma 2.6 that $\left[L_{a_{1}}^{\prime}, L_{a_{2}}, \ldots, L_{a_{n}}\right]$ is zero. Thus, we conclude that $L$ is nilpotent of class at most $n$.

Now suppose that the derived length of $L$ is at least 3. By the induction hypothesis, $[L, L]$ is nilpotent of bounded class. According to the previous paragraph, the quotient $L /[[L, L],[L, L]]$ is nilpotent of bounded class as well. Together, this gives nilpotency of $L$ of bounded class by the Lie ring analogue [1] of P. Hall's theorem [3]. The theorem is proved.

\section{MAin Results}

Although the condition (2.1) in the hypothesis of Theorem 2.13 may look somewhat artificial, we show in this section that some natural questions are reduced to the study of $\mathbb{Z} / p \mathbb{Z}$-graded Lie rings satisfying that condition. Thus, Theorem 2.13 seems to be an indispensable tool for handling the situations when a Frobenius group acts on another group or a Lie ring.

Theorem 3.1. Let FH be a Frobenius group with kernel $F$ of prime order and complement $H$ of order $q$. Assume that FH acts by automorphisms on a Lie ring 
$K$ in such a way that $C_{K}(F)=0$ and $C_{K}(H)$ is nilpotent of class $c$. Then $K$ is nilpotent of $(c, q)$-bounded class.

Proof. Let $F$ be of order $p$. Of course, by the Higman-Kreknin-Kostrikin theorem, $K$ is nilpotent of $p$-bounded class. Theorem 3.1 says that under the hypothesis the class can be bounded in terms of $c$ and $q$ only. Let $\omega$ be a $p$ th primitive root of 1 . We extend the ground ring by $\omega$ setting $\tilde{K}=K \otimes_{\mathbb{Z}} \mathbb{Z}[\omega]$. We view $\tilde{K}$ as a Lie algebra over $\mathbb{Z}[\omega]$. The group $F H$ acts in a natural way on $\tilde{K}$ and the action satisfies the conditions that $C_{\tilde{K}}(F)=0$ and $C_{\tilde{K}}(H)$ is nilpotent of class $c$. Let $\phi$ be a generator of $F$. For each $i=0, \ldots, p-1$ we define $L_{i}=\left\{x \in \tilde{K} \mid x^{\phi}=\omega^{i} x\right\}$. Set $L=\sum_{i} L_{i}$. Since $K$ admits a fixed-point-free automorphism of order $p$, there can be no $p$-torsion in $K$ (see, for example, [6, Corollary 1.7.3]). Hence $L$ is the direct sum of $L_{i}$, and so $L$ is naturally endowed with a $\mathbb{Z} / p \mathbb{Z}$-grading such that $L_{0}=0$. By [6. Lemma 4.1.1], $p \tilde{K} \leq L$. It follows that the nilpotency class of $L$ equals that of $\tilde{K}$ and therefore that of $K$. Indeed, suppose that $L$ is nilpotent of class $u$. Then $\gamma_{u+1}(p \tilde{K})=0$. Hence $p^{u+1} \gamma_{u+1}(\tilde{K})=0$. Since there is no $p$-torsion in $\tilde{K}$, we conclude that $\tilde{K}$ has nilpotency class $u$ and the same is true for $K$.

Thus, it is sufficient to show that $L$ is nilpotent of $(c, q)$-bounded class. We will deduce this from Theorem 2.13. Since $F$ is cyclic of prime order, $H$ is also cyclic. Let $H=\langle h\rangle, \phi^{h^{-1}}=\phi^{r}$. Then $r$ has multiplicative order $q$ modulo $p$. The group $H$ permutes the components $L_{i}$ so that $L_{i}{ }^{h}=L_{r i}$ for all $i \in \mathbb{Z} / p \mathbb{Z}$. Indeed, if $x_{i} \in L_{i}$, then $\left(x_{i}^{h}\right)^{\phi}=x_{i}^{h \phi h^{-1} h}=\left(x_{i}^{\phi^{r}}\right)^{h}=\omega^{i r} x_{i}^{h}$.

Given $u_{k} \in L_{k}$, we denote $u_{k}^{h^{i}}$ by $u_{r^{i} k}$. The sum over any $H$-orbit belongs to $C_{L}(H)$ and therefore

$$
u_{k}+u_{r k}+\cdots+u_{r^{q-1} k} \in C_{L}(H) .
$$

Let $x_{a_{1}}, \ldots, x_{a_{c+1}}$ be homogeneous elements in $L_{a_{1}}, \ldots, L_{a_{c+1}}$, respectively. Consider the elements

$$
\begin{aligned}
& X_{1}=x_{a_{1}}+x_{r a_{1}}+\cdots+x_{r^{q-1} a_{1}}, \\
& \vdots \\
& X_{c+1}=x_{a_{c+1}}+x_{r a_{c+1}}+\cdots+x_{r^{q-1} a_{c+1}} .
\end{aligned}
$$

Since all of them lie in $C_{L}(H)$ and since $C_{L}(H)$ is nilpotent of class $c$, it follows that

$$
\left[X_{1}, \ldots, X_{c+1}\right]=0 .
$$

After expanding the brackets, the left-hand side involves the term $\left[x_{a_{1}}, \ldots, x_{a_{c+1}}\right]$. Suppose that the commutator $\left[x_{a_{1}}, \ldots, x_{a_{c+1}}\right]$ is non-zero. Then there must be other non-zero terms in the expanded expression that belong to the same component $L_{a_{1}+\cdots+a_{c+1}}$. So there exist $i_{1}, \ldots, i_{m} \in\{1,2, \ldots, c+1\}$ and $\alpha_{1}, \ldots, \alpha_{m} \in$ $\{1,2, \ldots, q-1\}$ such that

$$
a_{i_{1}}+\cdots+a_{i_{m}}=r^{\alpha_{1}} a_{i_{1}}+\cdots+r^{\alpha_{m}} a_{i_{m}} .
$$

Thus, we have shown that $\left[x_{a_{1}}, \ldots, x_{a_{c+1}}\right]=0$ whenever the sequence $a_{1}, \ldots, a_{c+1}$ is $r$-independent. By Theorem 2.13 we conclude that $L$ is nilpotent of $(c, q)$-bounded class.

We will now embark on the proof of Theorem 1.1. Recall that for a group $G$ and a field $F$, a free $F G$-module of dimension $n$ is a direct sum of $n$ copies of the group 
algebra $F G$, each of which can be regarded as a vector space over $F$ of dimension $|G|$ with a basis $\left\{v_{g} \mid g \in G\right\}$ labeled by elements of $G$ on which $G$ acts in a regular representation: $v_{g} h=v_{g h}$. The next two lemmas were provided by Khukhro.

Lemma 3.2. Let $H$ be a soluble group of automorphisms of a finite group $G$, and $N$ an $H$-invariant elementary abelian p-subgroup of $G$ contained in the center $Z(G)$. If $N$, regarded as an $\mathbb{F}_{p} H$-module, is a free $\mathbb{F}_{p} H$-module, then $C_{G / N}(H)=$ $C_{G}(H) N / N$.

Proof. There is an easy induction on $|H|$ reducing to the case of $H$ of prime order. Indeed, if $H_{1}$ is a normal subgroup of $H$ of prime index $q$, then $N$ is also a free $\mathbb{F}_{p} H_{1}$-module. By the induction hypothesis, $C_{G / N}\left(H_{1}\right)=C_{G}\left(H_{1}\right) N / N$. Since the centralizer of $H$ in any section is contained in the centralizer of $H_{1}$ in that section, we can replace $G$ by $C_{G}\left(H_{1}\right)$. Then $N_{1}=C_{N}\left(H_{1}\right)=C_{G}\left(H_{1}\right) \cap N$ is a free $\mathbb{F}_{p}\left(H / H_{1}\right)$-module. If we prove the lemma for the group $H / H_{1}$ of prime order $q$ acting on $C_{G}\left(H_{1}\right)$, then

$$
\begin{aligned}
C_{G / N}(H) & =C_{C_{G / N}\left(H_{1}\right)}\left(H / H_{1}\right)=C_{C_{G}\left(H_{1}\right) N / N}\left(H / H_{1}\right) \\
& \cong C_{C_{G}\left(H_{1}\right) /\left(C_{G}\left(H_{1}\right) \cap N\right)}\left(H / H_{1}\right) \\
& =C_{C_{G}\left(H_{1}\right)}\left(H / H_{1}\right)\left(C_{G}\left(H_{1}\right) \cap N\right) /\left(C_{G}\left(H_{1}\right) \cap N\right) \\
& =C_{G}(H)\left(C_{G}\left(H_{1}\right) \cap N\right) /\left(C_{G}\left(H_{1}\right) \cap N\right) \cong C_{G}(H) /\left(C_{G}(H) \cap C_{N}\left(H_{1}\right)\right) \\
& =C_{G}(H) / C_{N}(H)=C_{G}(H) /\left(C_{G}(H) \cap N\right) \cong C_{G}(H) N / N .
\end{aligned}
$$

Thus, we can assume from the outset that $H=\langle h\rangle$ is cyclic of prime order $q$. The following calculation was in fact already performed in [5], but we reproduce it here for the benefit of the reader. Although it can be carried out for any dimension of the $\mathbb{F}_{p} H$-module $N$, it is easier to use induction on this dimension.

First suppose that $N$ is a one-dimensional free $\mathbb{F}_{p} H$-module; that is, in additive notation, $N$, as an $\mathbb{F}_{p}$-space, has a basis $e_{1}, \ldots, e_{q}$, which is cyclically permuted by $h: e_{1} h=e_{2}, \ldots, e_{q-1} h=e_{q}, e_{q} h=e_{1}$. Let $c+N \in C_{G / N}(h)$ (we use additive notation working in the abelian group $\langle c\rangle+N)$. Then $c h=c+\left(k_{1} e_{1}+\cdots+k_{q} e_{q}\right)$ for some $k_{i} \in \mathbb{F}_{p}$ and we seek a fixed point in $G$ in the form $c+\left(x_{1} e_{1}+\cdots+x_{q} e_{q}\right)$. We need to solve the equation

$$
c+\left(x_{1} e_{1}+\cdots+x_{q} e_{q}\right)=\left(c+\left(x_{1} e_{1}+\cdots+x_{q} e_{q}\right)\right) h,
$$

which gives

$$
c+\left(x_{1} e_{1}+\cdots+x_{q} e_{q}\right)=c+\left(k_{1} e_{1}+\cdots+k_{q} e_{q}\right)+\left(x_{1} e_{2}+\cdots+x_{q-1} e_{q}+x_{q} e_{1}\right)
$$

or

$$
\left(x_{1}-x_{q}\right) e_{1}+\left(x_{2}-x_{1}\right) e_{2}+\cdots+\left(x_{q}-x_{q-1}\right) e_{1}=k_{1} e_{1}+\cdots+k_{q} e_{q} .
$$

Since the $e_{i}$ form a basis, this is equivalent to the linear system over $\mathbb{F}_{p}$ for the unknowns $x_{i}$,

$$
\left(\begin{array}{cccc}
1 & & & -1 \\
-1 & 1 & & \\
& \ddots & \ddots & \\
& & -1 & 1
\end{array}\right)\left(\begin{array}{c}
x_{1} \\
x_{2} \\
\vdots \\
x_{q}
\end{array}\right)=\left(\begin{array}{c}
k_{1} \\
k_{2} \\
\vdots \\
k_{q}
\end{array}\right)
$$

The rank of the $q \times q$ coefficient matrix is $q-1$, with the non-trivial dependence being zero sum of rows. But we also have $\sum_{i=1}^{q} k_{i}=0$, because $h^{q}=1$. Hence the system has a solution, as required. 
Now suppose that $N$ has a proper one-dimensional free $\mathbb{F}_{p} H$-submodule $N_{1}$; then $N / N_{1}$ is also a free $\mathbb{F}_{p} H$-module of smaller dimension than $N$. By induction, $C_{G / N}(H)=\left(C_{G / N_{1}}(H) N / N_{1}\right) /\left(N / N_{1}\right)$, and by the one-dimensional case, this is equal to

$$
\left(\left(C_{G}(H) N_{1} / N_{1}\right) N / N_{1}\right) /\left(N / N_{1}\right) \cong C_{G}(H) N / N
$$

as required.

Lemma 3.3. Let $G F H$ be a double Frobenius group, where $G$ is a normal subgroup, $G F$ is a Frobenius group with kernel $G$ and complement $F$, and $F H$ is a Frobenius group with kernel $F$ and complement $H$. Then

(a) for every term of the lower central series $\gamma_{i}=\gamma_{i}(G)$ we have $C_{G / \gamma_{i}}(H)=$ $C_{G}(H) \gamma_{i} / \gamma_{i}$

(b) the nilpotency class of the fixed-point subring $C_{L(G)}(H)$ for the induced group of automorphisms $H$ does not exceed the nilpotency class of the centralizer $C_{G}(H)$.

Proof. (a) As a Frobenius complement in $G F$, the group $F$ has no non-cyclic subgroups of order $p^{2}$ for any prime $p$. Hence the Sylow subgroups of $F$ are cyclic or generalized quaternion. Being the Frobenius kernel of $F H$, the group $F$ is nilpotent. It follows that the Sylow 2-subgroup of $F$ is trivial, for otherwise its center would contain a non-trivial fixed point for $H$. Thus, $F$ is cyclic. Let $F_{1}$ be a subgroup of $F$ of prime order $p$. The hypothesis of the theorem is inherited by $G F_{1} H$. Therefore we can replace $F$ by $F_{1}$ and assume that $F$ has prime order $p$. Therefore $H$ is cyclic, say, of order $q$. Consider an unrefinable $F H$-invariant central series

$$
G>\gamma_{i}=N_{1}>N_{2}>\cdots>N_{k}>N_{k+1}=1
$$

with elementary abelian factors $N_{i} / N_{i+1}$ connecting $\gamma_{i}$ with 1 . Each factor $V$ of this series can be regarded as an irreducible $\mathbb{F}_{r} F H$-module for a suitable prime $r$.

We claim that $V$ is a free $\mathbb{F}_{r} H$-module. Indeed, after we adjoin a primitive $p$ th root of unity $\omega$, the resulting $\mathbb{F}_{r}(\omega) F H$-module $\widetilde{V}$ decomposes into a direct sum of irreducible $\mathbb{F}_{r}(\omega) F H$-modules; let $W$ be any of them. Since $G F$ is a Frobenius group, $F$ has no fixed points on any section of $G$ and therefore acts faithfully on $W$. Hence so does $F H$, as $[F, g]=F$ for any $g \in H$. By Clifford's theorem, or more precisely, by Theorem 3.4.3 in [2], the number of Wedderburn components of $W$ with respect to $F$ is exactly $|H|=q$, and $H$ cyclically permutes these components. Hence $W$ is a free $\mathbb{F}_{r}(\omega) H$-module and therefore $\widetilde{V}$ is also a free $\mathbb{F}_{r}(\omega) H$-module. In other words, there is a basis of $\widetilde{V}$ as a vector space over $\mathbb{F}_{r}(\omega)$ in which the generator $h$ of $H$ is represented by a block-diagonal matrix with $q \times q$ blocks

$$
\left(\begin{array}{ccccc}
0 & 1 & & & \\
& 0 & 1 & & \\
& & 0 & \ddots & \\
& & & \ddots & 1 \\
1 & & & & 0
\end{array}\right) .
$$

But this matrix is defined over $\mathbb{F}_{r}$. Since the matrix of $h$ in some basis of $V$ (which is also a basis of $\widetilde{V}$ ) is similar over $\mathbb{F}_{r}(\omega)$ to that matrix, the matrix of $h$ is also similar over $\mathbb{F}_{r}$ to the same matrix. In other words, there is a basis of $V$ in which the matrix of $h$ has the same block-diagonal form. Hence $V$ is a free $\mathbb{F}_{r} H$-module. 
We can now apply Lemma 3.2 in an easy induction on the length of the series (3.1) to find an element of $C_{G}(H)$ in any $g N_{1}=g \gamma_{i} \in C_{G / \gamma_{i}}(H)$. For $k>1$ there is $c_{1} N_{k} \in C_{G / N_{k}}(H) \cap g N_{1} / N_{k}$. By Lemma 3.2 applied with $N=N_{k}$, there is a required $c \in C_{G}(H) \cap c_{1} N_{k} \subseteq C_{G}(H) \cap g N_{1}$.

(b) By the definition of the associated Lie ring,

$$
C_{L(G)}(H)=\bigoplus_{i} C_{\gamma_{i} / \gamma_{i+1}}(H),
$$

which by part (a) is equal to

$$
\bigoplus_{i} C_{\gamma_{i}}(H) \gamma_{i+1} / \gamma_{i+1}
$$

The Lie ring products in $L(G)$ are defined for elements of the $\gamma_{i} / \gamma_{i+1}$ in terms of the images of the group commutators and then extended by linearity. Let $c$ be the nilpotency class of $C_{G}(H)$; then any group commutator of weight $c+1$ in elements of $C_{G}(H)$ is trivial; hence any Lie ring commutator of weight $c+1$ in elements of $C_{\gamma_{i}}(H) \gamma_{i+1} / \gamma_{i+1}$ is also trivial. Since these elements generate $C_{L(G)}(H)$, this subring is nilpotent of class at most $c$.

Now we are ready to prove Theorem 1.1 .

Let $G F H$ be a double Frobenius group with complement $H$ of order $q$. Assume that $C_{G}(H)$ is nilpotent of class $c$. Then $G$ is nilpotent of $(c, q)$-bounded class.

Proof. Note that the subgroup $G$ is nilpotent of class bounded in terms of the least prime divisor of the order of $F$. But our task is to bound the nilpotency class of $G$ in terms of $c$ and $q$. Arguing as in Lemma 3.3 we can assume that $F$ has prime order $p$.

Let $L(G)$ be the associated Lie ring of the group $G$. Thus,

$$
L(G)=\bigoplus_{i=1}^{n} \gamma_{i} / \gamma_{i+1},
$$

where $n$ is the nilpotency class of $G$ and the $\gamma_{i}$ are the terms of the lower central series of $G$. The nilpotency class of $G$ coincides with that of $L(G)$. The action of the group $F H$ on $G$ naturally induces an action of $F H$ on $L(G)$. Because $F$ acts fixed-point-freely on every quotient $\gamma_{i} / \gamma_{i+1}$ [2, Lemma 10.1.3], it follows that $C_{L(G)}(F)=0$. Lemma 3.3 tells us that $C_{L(G)}(H)$ is nilpotent of class at most $c$. We now deduce from Theorem 3.1 that $L(G)$ is nilpotent of $(c, q)$-bounded class. Since the class of $G$ is equal to that of $L(G)$, the result follows.

\section{REFERENCES}

1. Chong-Yun Chao, Some characterizations of nilpotent Lie algebras, Math. Z. 103 (1968), 40-42. MR 0223415 (36:6463)

2. D. Gorenstein, Finite groups, Harper and Row, New York, 1968. MR.0231903 (38:229)

3. P. Hall, Some sufficient conditions for a group to be nilpotent. Ill. J. Math. 2 (1958), 787-801. MR0105441 (21:4183)

4. G. Higman, Groups and rings which have automorphisms without non-trivial fixed elements. J. London Math. Soc. (2) 32 (1957), 321-334. MR0089204 (19:633c)

5. E. I. Khukhro, Fixed points of p-automorphisms of finite p-groups. Algebra i Logika 14 (1975), 697-703; English transl., Algebra and Logic 14 (1976), 417-420. MR0427472 (55:504)

6. E. I. Khukhro, Nilpotent groups and their automorphisms, Walter de Gruyter, Berlin, New York, 1993. MR1224233 (94g:20046) 
7. E. I. Khukhro, Graded Lie rings with many commuting components and an application to 2-Frobenius groups, Bull. London Math. Soc. 40 (2008), 907-912. MR2439656 (2009m:17029)

8. E. I. Khukhro and V. D. Mazurov (Eds.), Unsolved Problems in Group Theory. The Kourovka Notebook, no. 17, Institute of Mathematics, Novosibirsk, 2010.

9. V. A. Kreknin, The solvability of Lie algebras with regular automorphisms of finite period, Math. USSR Doklady 4 (1963), 683-685. MR0157990 (28:1218)

10. V. A. Kreknin and A. I. Kostrikin, Lie algebras with regular automorphisms, Math. USSR Doklady 4 (1963), 355-358. MR0146230 (26:3752)

11. V. D. Mazurov, Recognition of the finite simple groups $S_{4}(q)$ by their element orders, Algebra and Logic 41 (2002), 93-110. MR:1922988 (2003e:20018)

12. A. Shalev, Automorphisms of finite groups of bounded rank, Israel J. Math. 82 (1993), 395404. MR1239058 (95b:20038)

13. J. G. Thompson, Finite groups with fixed-point-free automorphisms of prime order, Proc. Nat. Acad. Sci. USA 45 (1959), 578-581. MR.0104731(21:3484)

Sobolev Institute of Mathematics, Novosibirsk, 630090, Russia

Current address: Laboratoire de Mathématiques, Informatique et Application, Université de Haute Alsace, Mulhouse, 68093, France

E-mail address: natalia_makarenko@yahoo.fr

Department of Mathematics, University of Brasilia, Brasilia-DF, 70910-900, Brazil

E-mail address: pavel@mat.unb.br 